\title{
Transcutaneous Bilirubin Measurements Can Be Used to Measure Bilirubin Levels during Phototherapy
}

\author{
Saad Abdullah Alsaedi (iD \\ Department of Pediatrics, Faculty of Medicine, King Abdulaziz University, Jeddah, Saudi Arabia \\ Correspondence should be addressed to Saad Abdullah Alsaedi; salsaedi@hotmail.com
}

Received 3 November 2017; Revised 26 January 2018; Accepted 31 January 2018; Published 20 March 2018

Academic Editor: Francesco Porta

Copyright (C) 2018 Saad Abdullah Alsaedi. This is an open access article distributed under the Creative Commons Attribution License, which permits unrestricted use, distribution, and reproduction in any medium, provided the original work is properly cited.

\begin{abstract}
Objective. To determine whether transcutaneous bilirubin measurements (TcB) before and during phototherapy taken from covered skin during phototherapy correlate with total serum bilirubin (TSB) levels. Study Design. In this prospective observational study, healthy term newborns who required TSB measurements were included. TcB measurements were taken from the forehead before starting and during phototherapy using the BiliChek device. Before starting phototherapy, part of the forehead was covered. Blood for TSB measurement was collected within 5 minutes of TcB measurements. Correlations and mean differences between TcB and TSB before and during phototherapy were calculated. Result. Paired TSB and TcB measurements before and during phototherapy in 151 newborns were performed. The mean gestational age was 38.8 weeks and birth weight was $3.1 \mathrm{~kg} ; 53 \%$ were male. Before starting phototherapy, TSB and TcB were $183.8 \pm 41.6$ and $190.5 \pm 43 \mu \mathrm{mol} / \mathrm{l}$, respectively. During phototherapy, TSB and TcB were $191.8 \pm 39.4$ and $187.8 \pm 45.3 \mu \mathrm{mol} / \mathrm{l}$, respectively. Linear regression analysis showed a significant correlation between TcB and TSB before starting phototherapy and during phototherapy $(r: 0.85 ; p<0.001$ and $r: 80.0 ; p<0.001)$, respectively. Before starting phototherapy, the mean difference between TSB and TcB was $6.2 \pm 23.2 \mu \mathrm{mol} / \mathrm{l}$, with a $95 \%$ CI of -39.3 to $51.7 \mu \mathrm{mol}$. During phototherapy, the mean difference was $-2.8 \pm 23.5 \mu \mathrm{mol} / 1$, with a $95 \% \mathrm{CI}$ of -48.9 to $43.3 \mu \mathrm{mol} / \mathrm{l}$. Conclusion. TcB measurements from covered skin in jaundiced term infants during phototherapy correlate with TSB and can be used to monitor bilirubin levels during phototherapy.
\end{abstract}

\section{Introduction}

Hyperbilirubinemia is common during the neonatal period and sometime requires treatment with either phototherapy or exchange transfusion. Management of neonatal hyperbilirubinemia becomes more challenging when at-risk newborn infants are discharged early without appropriate postdischarge follow-up. This increases the risk of severe hyperbilirubinemia and its complications such as acute bilirubin encephalopathy, and bilirubin-induced neurologic dysfunction (BIND) in these infants [1]. Although the measurement of TSB is still the standard of care in the assessment of neonatal hyperbilirubinemia, it requires venous or heel prick blood samples, which is an invasive and painful procedure. Transcutaneous bilirubin (TcB) measurement is an easy, painless, and timesaving alternative to TSB measurement $[2,3]$. Several studies have shown a good correlation between
TSB and TcB measurements in term and late preterm infants $[3,4]$. TcB measurement is an essential part of screening for neonatal jaundice that can decrease the need for TSB $[4,5]$. Jaundiced newborn infants under phototherapy require close monitoring of TSB, which means frequent blood sampling. In addition, there has been an increasing trend towards home phototherapy in the low-risk term and near-term infants, where follow-up assessments of TSB are cumbersome [6-8]. Such infants can benefit from TcB measurement at home if validated. Bilirubin in the skin exposed to phototherapy is modified and significantly affects TcB. Phototherapy converts the bilirubin to the more water-soluble lumirubin, which is excreted by the body. This process causes blanching of the skin, which obviously will change TcB levels. However, the accuracy of $\mathrm{TcB}$ measurement during phototherapy is still not clear. Some studies reported that, by covering the skin during phototherapy, more accurate approximations of 
TSB could be made with $\mathrm{TcB}$ measurement though TcB measurement tends to underestimate TSB when bilirubin levels are $>214 \mu \mathrm{mol} / \mathrm{L}$ [6]. However, other studies reported insignificant difference between TcB and TSB measurements $[9,10]$. The sample size of some of these studies was small and the study populations were not homogeneous where both term and preterm newborns were included. The aim of this study was to evaluate the accuracy of TcB measurement from a shielded area at the forehead of jaundiced term newborns during treatment with phototherapy.

\section{Methods}

2.1. Subjects. This was a prospective observational study conducted at the newborn nursery of a tertiary care center with 4000 deliveries per year during the period of January 2015 to December 2016. We conducted the study according to the principles of Helsinki Declaration. The Institutional Review Board approved the study. We obtained informed consent from the parents of the newborn infants before inclusion in the study. We included healthy term newborns who required phototherapy for management of neonatal hyperbilirubinemia as per the decision of the treating physician. We excluded infants who had conjugated hyperbilirubinemia, major congenital malformation, sepsis, or congenital viral infections.

2.2. Protocol. During the study period, we measured TcB in all jaundiced, otherwise healthy, term newborns who required TSB measurements. The attending neonatologist made the decision when to start continuous phototherapy based on the American Academy of Pediatrics guideline [5] as well as the frequency of obtaining TSB measurements. In our nursery, we use a standard phototherapy unit (Photo Therapy 4000, Draeger Medical Telford, PA, USA). The total power irradiance during phototherapy was $28-30 \mathrm{~W} / \mathrm{cm}^{2} / \mathrm{nm}$. We included in this study healthy term newborns who required phototherapy. Both eyes were protected from phototherapy light with eye patches (Biliband, Natus Medical Incorporated, Seattle, WA, USA). We measured TcB before and during phototherapy from the same area in the middle of the forehead. We avoided areas with hair, hyperpigmentation, bruises, and hemangioma. Before starting phototherapy, we covered a portion at the middle of the forehead, by a photoopaque patch $2.5 \mathrm{~cm}$ in diameter (BilEclipse Phototherapy Patch, Respironics, Murrysville, PA, USA) to shield it from light exposure. We used a single BiliChek ${ }^{\circledR}$ device to measure $\mathrm{TcB}$. We measured all TcB from the covered part of the forehead, while the infant is under phototherapy within 5 minutes of drawing blood for TSB measurements. Once phototherapy was started, we repeated the TcB measurement with the first TSB. We turned off the phototherapy during TcB measurements and blood sampling for TSB. We calibrated the BiliChek before each measurement using a disposable probe (BiliCal, SpectRx, Norcross, GA, USA) as per manufacturer's instruction [11]. BiliChek displays the average bilirubin in micromole/l of 5 measurements for each TcB. BiliChek was in use in our nursery to measure TcB for the last 5 years, so all the nurses in the nursery were
TABLE 1: Total serum and transcutaneous bilirubin levels before and during phototherapy in umol/l.

\begin{tabular}{lcc}
\hline Bilirubin & Mean \pm SD & Range \\
\hline TSB before phototherapy & $183.8 \pm 41.6$ & $152-314.5$ \\
TcB before phototherapy & $190.5 \pm 43$ & $147-296$ \\
Mean & $187 \pm 32$ & $175-300$ \\
Difference & $6.2 \pm 23.2^{*}$ & $-5.2-51$ \\
TSB during phototherapy & $191.8 \pm 39.4$ & $177-278.8$ \\
TcB during phototherapy & $187.8 \pm 45.3$ & $160-311.1$ \\
Mean & $186.9 \pm 44.9$ & $170-287.3$ \\
Difference & $-2.8 \pm 23.5^{*}$ & $-86.7-85$ \\
\hline$*$
\end{tabular}

${ }^{*} p$ value $=0.2$.

trained in its use. However, for the purpose of the study, 5 nurses performed all TcB measurements. We obtained all blood specimens by heel stick. We collected the blood by drip method into heparin-containing tubes. We sent the blood specimen to the laboratory immediately for analysis using Diazo method (Dimension Vist ${ }^{\circledR}$ System and Flex ${ }^{\circledR}$ reagent cartridge, Siemens) [12].

We recorded the following demographic data for all newborns enrolled in the study: gestational age, mode of delivery, sex, birth weight, etiology of jaundice, and postnatal age in hours at the time of TSB and TcB measurements.

2.3. Statistical Analysis. We used the Statistical Package for the Social Sciences (version 21, Chicago, IL, USA) for statistical analysis. We used Pearson's correlation and linear regression model including 95\% confidence interval (CI) to assess the agreement between TSB and TcB before and during phototherapy. Pearson's coefficient alone can be a poor indicator to estimate the agreement between two diagnostic tests, so we used the Bland-Altman analysis to assess TSB and $\mathrm{TcB}$ variability [12]. In this analysis, the mean bias of TCB - TSB was compared against their mean for every patient and variability was defined as \pm 1.96 standard deviation of the mean bias. Because some studies reported that TcB underestimates TSB at levels $>214 \mu \mathrm{mol} / \mathrm{L}$, we calculated the mean biases before and during phototherapy at TSB levels $<214 \mu \mathrm{mol} / \mathrm{L}$ and $>214 \mu \mathrm{mol} / \mathrm{L}$.

\section{Results}

We performed TSB and TcB measurements before and during phototherapy in 151 healthy term infants. The mean gestational age was $38.8 \pm 1.2$ weeks (range; $37-41$ weeks), mean birth weight $(\mathrm{BW})$ was $3.1 \pm 0.6 \mathrm{~kg}$ (range $1.8-5.2 \mathrm{~kg}$ ), $80(53 \%)$ were male, and $116(77 \%)$ were born by spontaneous vaginal delivery. The causes of jaundice were physiological jaundice in $62(41 \%)$ newborns, $\mathrm{ABO}$ incompatibility in $45(30 \%)$, Rh incompatibility in 10 (6.6\%), G6PD in 14 (9.3\%), polycythemia in $13(8.6 \%)$, and cephalohematoma in 7 (4.6\%). The means of TSB, TcB, and difference between $\mathrm{TcB}$ and TSB are shown in Table 1. Linear regression analysis showed a significant correlation between TcB and TSB before starting phototherapy (Figure 1(a)) and during phototherapy 


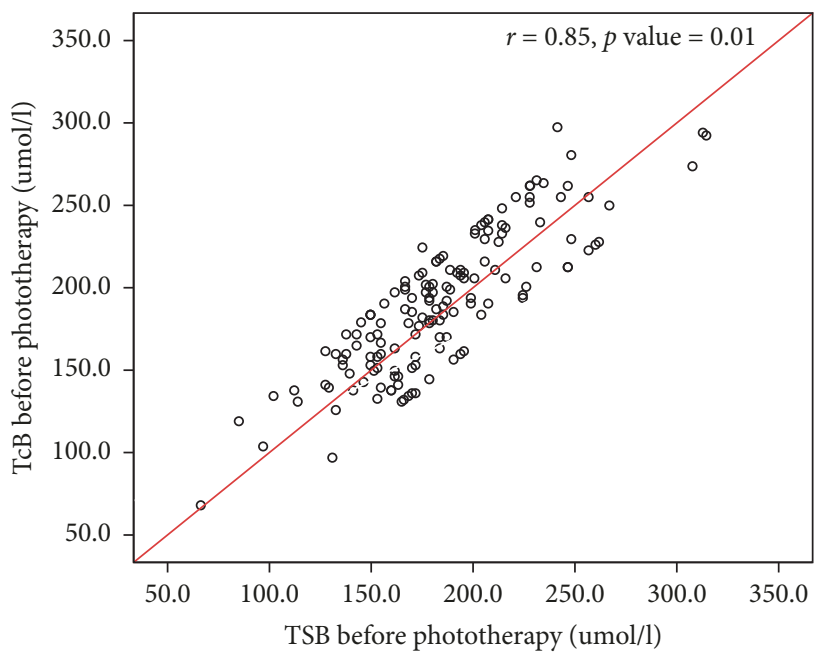

(a)

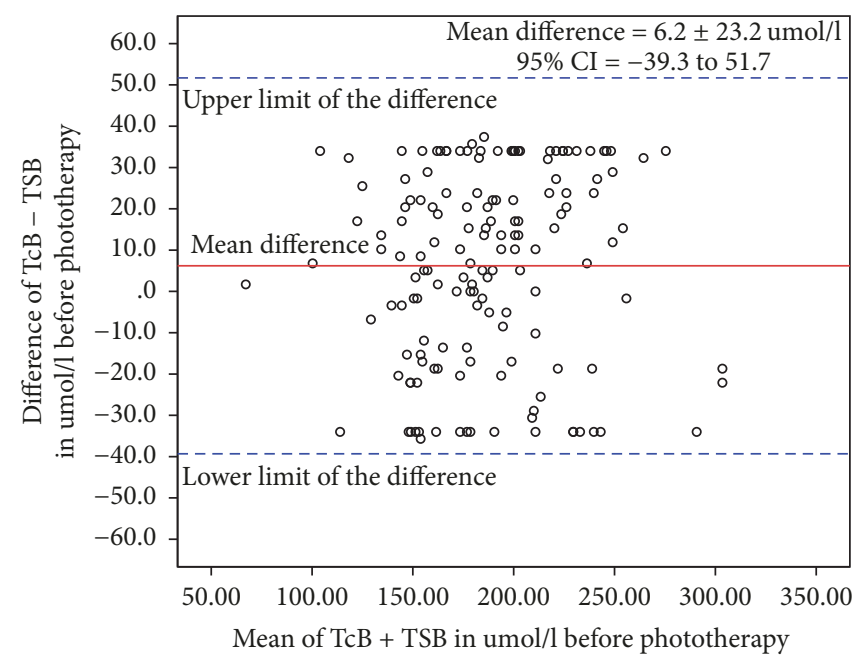

(b)

FIGURE 1: (a) Relationship between transcutaneous bilirubin (TcB) and total serum bilirubin (TSB) levels before starting phototherapy. (b) Bland-Atman Plot of transcutaneous bilirubin and total serum bilirubin values before starting phototherapy.

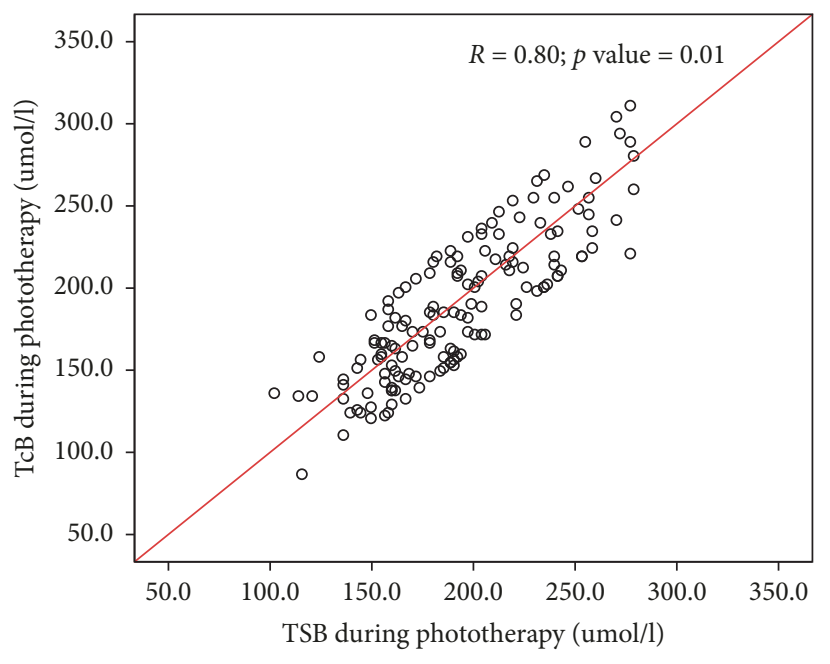

(a)

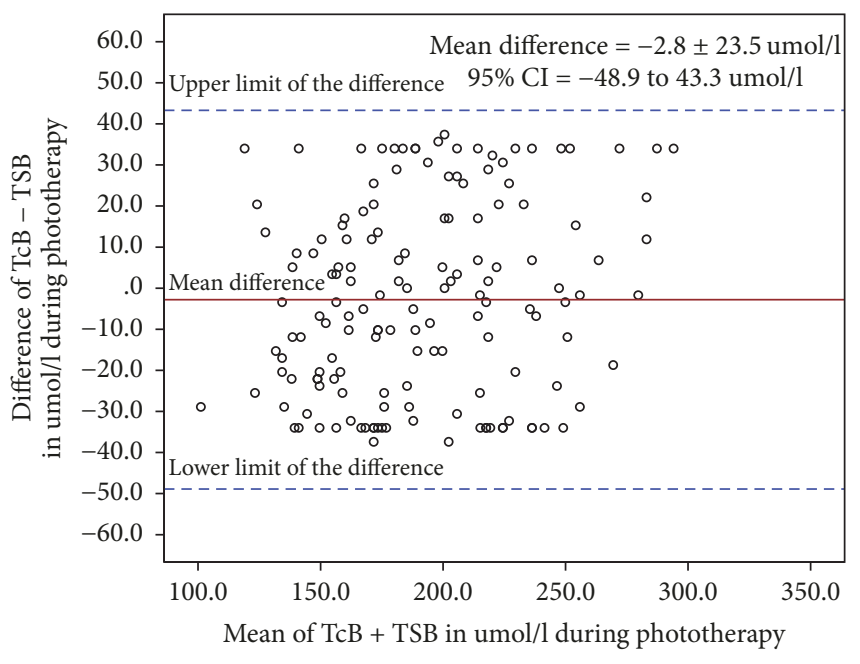

(b)

Figure 2: (a) Relationships between transcutaneous bilirubin (TcB) and total serum bilirubin (TSB) levels during phototherapy. (b) BlandAtman Plot of transcutaneous bilirubin and total serum bilirubin values during phototherapy.

(Figure 2(a)) $(r: 0.85 ; p<0.001$, and $r: 0.80 ; p<0.001)$, respectively. The Bland-Altman plot demonstrates the level of precision of the BiliChek device by comparing the difference versus the average of measurements between TcB and TSB values before (Figure 1(b)) and during (Figure 2(b)) phototherapy. Before starting phototherapy, the mean difference between these measurements was $6.2 \pm 23.2 \mu \mathrm{mol} / 1$ with a $95 \%$ CI of -39.3 to $51.7 \mu \mathrm{mol} / 1$. During phototherapy, the mean difference was $-2.8 \pm 23.5 \mu \mathrm{mol} / \mathrm{l}$ with a $95 \% \mathrm{CI}$ of -48.9 to $43.3 \mu \mathrm{mol} / \mathrm{l}$. During phototherapy, TcB measurements underestimated TSB in 53 (35\%) infants. TcB underestimated TSB by $\leq 25.5 \mu \mathrm{mol} / \mathrm{l}$ in $33(21.8 \%)$ infants, by $>25.5-\leq 34 \mu \mathrm{mol} / \mathrm{l}$ in $19(12.5 \%)$ infants, and by $>34-51 \mu \mathrm{mol} / 1$ in $1(0.7 \%)$ infant. TSB levels were $<214 \mu \mathrm{mol} / \mathrm{L}$ in 111 (73\%) newborns. There was a significant correlation between TcB and TSB before starting phototherapy and during phototherapy $(r=0.87$; $p<0.01$, and $r=0.82 ; p<0.01$ ), respectively. Before starting phototherapy, the mean difference between TSB and TcB was $10.4 \pm 28.1 \mu \mathrm{mol} / \mathrm{l}, 95 \% \mathrm{CI}=-44.7-65.5 \mu \mathrm{mol} / \mathrm{l}$. During phototherapy, the mean difference between TSB and $\mathrm{TcB}$ was $-3.7 \pm 29.3 \mu \mathrm{mol} / \mathrm{l}, 95 \% \mathrm{CI}=-60.9-53 \mu \mathrm{mol} / \mathrm{l}$. TSB levels were $>214 \mu \mathrm{mol} / \mathrm{L}$ in 40 (27\%) newborns. There was a significant correlation between TcB and TSB before starting phototherapy and during phototherapy $(r=0.80$; $p<0.02$, and $r=0.77 ; p<0.04)$, respectively. Before starting phototherapy, the mean difference between these measurements was $-4.5 \pm 39.5 \mu \mathrm{mol} / \mathrm{l}, 95 \% \mathrm{CI}=$ $-81.9-72.9 \mu \mathrm{mol} / 1$. During phototherapy, the mean difference 
between these measurements was $-3.6 \pm 37.4 \mu \mathrm{mol} / 1,95 \% \mathrm{CI}$ $=-76.9-69.7 \mu \mathrm{mol} / 1$.

\section{Discussion}

Our study showed a good correlation between TcB as measured from a shielded area from phototherapy at the forehead and TSB measurements in a cohort of jaundiced term newborns during phototherapy. The correlations of $\mathrm{TcB}$ and TSB before and during phototherapy were high and comparable. There was no significant difference between TSB and TcB from covered area of the skin. The correlation between TSB and TcB was significant before and during phototherapy when TSB levels were $>214 \mu \mathrm{mol} / \mathrm{l}$, but less than that when TSB levels $<214 \mu \mathrm{mol} / \mathrm{l}$. The Bland-Atman analysis demonstrated that $\mathrm{TcB}$ measurements during phototherapy underestimate TSB (negative mean bias) regardless of TSB levels and there was a wide limit of agreement between $\mathrm{TcB}$ and TSB, which worsened as TSB levels increase. In contrast, $\mathrm{TCB}$ measurements before starting phototherapy overestimate TSB. The underestimation of TcB to TSB levels by $>25.5-51 \mu \mathrm{mol} / 1$ in $13.2 \%$ of the studied infants led us to recommend that while the infant is under phototherapy a confirmatory TSB measurement should be done when TcB level is $\leq 51 \mu \mathrm{mol} / \mathrm{L}$ below the bilirubin level at which phototherapy should be discontinued. Reyes et al. [6] reported that $\mathrm{TcB}$ measured by BiliChek during phototherapy compared to TSB has a negative mean bias which worsened as TSB increased particularly at TSB levels $>238 \mu \mathrm{mol} / \mathrm{l}$. Katayama et al. [13] reported moderate correlations between TSB and TcB before $(r=0.56)$ and during $(r=0.47)$ phototherapy. For TSB $\leq 306 \mu \mathrm{mol} / \mathrm{L}$ during phototherapy, a TcB cut-off of $238 \mu \mathrm{mol} / 1$ had a specificity of $100 \%$. Zecca et al. [9] and Fonseca et al. [10] reported a good agreement between TSB and $\mathrm{TcB}$ measured from patched area of the skin, while TcB measurement from unpatched skin underestimates TSB levels. However, Murli et al. [14] found poor agreement between TcB and STB before and during phototherapy in late preterm and term newborns. Different study populations, inclusion of preterm and term newborns in some studies, and different irradiance of phototherapy light can explain these variations in the results of these studies.

$\mathrm{TcB}$ measurement in jaundiced, healthy otherwise, full term infants under phototherapy from properly shielded area of the skin is relatively accurate and can be performed to monitor bilirubin levels. It will lead to a decrease in the frequency of painful blood sampling. However, TcB measurement should not be considered a surrogate for the gold standard TSB measurement. If the infant is found to have significant hyperbilirubinemia, a confirmatory TSB should be considered.

The strength of our study includes a prospective study with a relatively good sample size. We included only homogeneous healthy, term infants rather than including mixed population of term and preterm infants. However, our study has some limitations. First, we obtained TcB during phototherapy at $8 \pm 2$ hours after starting phototherapy, so we do not know if the same correlation between TSB and TcB will exist if we measure $\mathrm{TcB}$ during longer intervals, while the infants are still under phototherapy. Second, trained nurses performed measurements of $\mathrm{TcB}$ rather than a specific person whether a physician or nurse and this raises the issue of user variability. However, in practice, nurses perform TcB measurements. As with any point of care test, assessment of the competency of the personnel using TcB device is very important.

Future studies should address the correlation of TcB and TSB at specific intervals during the entire time of phototherapy rather than one paired TcB and TSB measurement. These studies should also address the number of painful heel sticks which will be reduced when $\mathrm{TcB}$ device is used to monitor the bilirubin levels during phototherapy treatment as well as the cost effectiveness.

In conclusion, our study supports previous studies which concluded that $\mathrm{TcB}$ measurements taken from a covered area of the skin in jaundiced, healthy, full term infants under phototherapy correlate significantly with TSB and can be used to monitor bilirubin levels during phototherapy.

\section{Conflicts of Interest}

The author declares no conflicts of interest.

\section{Acknowledgments}

This study could have not been possible without the help of the nurses of the newborn nursery and the parents of the newborns who consented for involvement of their babies in this study.

\section{References}

[1] M. J. Maisels and T. B. Newman, "Jaundice in full-term and near-term babies who leave the hospital within 36 h," Clinics in Perinatology, vol. 25, pp. 295-302, 1998.

[2] F. F. Rubaltelli, G. R. Gourley, N. Loskamp et al., "Transcutaneous bilirubin measurement: A multicenter evaluation of a new device," Pediatrics, vol. 107, no. 6, pp. 1264-1271, 2001.

[3] M. J. Maisels, E. M. Ostrea Jr., S. Touch et al., "Evaluation of a new transcutaneous bilirubinometer," Pediatrics, vol. 113, no. 6 , pp. 1628-1635, 2004.

[4] S. Alsaedi, "Transcutaneous bilirubin measurement in healthy Saudi term newborns," Saudi Medical Journal, vol. 37, pp. 179183, 2016.

[5] American Academy of Pediatrics Subcommittee on Hyperbilirubinemia, "Management of hyperbilirubinemia in the newborn infant 35 or more weeks of gestation," Pediatrics, vol. 114, no. 1, pp. 297-316, 2004.

[6] C. A. Reyes, D. R. Stednitz, C. Hahn, K. D. Mutchie, S. R. McCullough, and K. Kronberg, "Evaluation of the BiliChek being used on hyperbilirubinemic newborns undergoing home phototherapy," Archives of Pathology \& Laboratory Medicine, vol. 132, no. 4, pp. 684-689, 2008.

[7] M. Walls, A. Wright, P. Fowlie, and L. Irvine, "Home phototherapy in the United Kingdom," Archives of Disease in Childhood: Fetal and Neonatal Edition, vol. 89, p. F282, 2004.

[8] K. Zainab and S. Adlina, "Effectiveness of home versus hospital phototherapy for term infants with uncomplicated hyperbilirubinemia: A pilot study in Pahang, Malaysia," Medical Journal of Malaysia, vol. 59, no. 3, pp. 395-401, 2004. 
[9] E. Zecca, G. Barone, D. De Luca, R. Marra, E. Tiberi, and C. Romagnoli, "Skin bilirubin measurement during phototherapy in preterm and term newborn infants," Early Human Development, vol. 85, no. 8, pp. 537-540, 2009.

[10] R. Fonseca, R. Kyralessa, M. Malloy, J. Richardson, and S. K. Jain, "Covered skin transcutaneous bilirubin estimation is comparable with serum bilirubin during and after phototherapy," Journal of Perinatology, vol. 32, no. 2, pp. 129-131, 2012.

[11] C. Romagnoli, E. Zecca, P. Catenazzi, G. Barone, and A. A. Zuppa, "Transcutaneous bilirubin measurement: Comparison of Respironics BiliCheck and JM-103 in a normal newborn population," Clinical Biochemistry, vol. 45, no. 9, pp. 659-662, 2012.

[12] D. Giavarina, "Understanding Bland Altman analysis," Biochemia Medica, vol. 25, no. 2, pp. 141-151, 2015.

[13] Y. Katayama, M. Enomoto, S. Kikuchi et al., "Transcutaneous bilirubin measurement during phototherapy in term neonates," Pediatrics International, vol. 59, no. 6, pp. 686-690, 2017.

[14] L. Murli, A. Thukral, M. J. Sankar et al., "Reliability of transcutaneous bilirubinometry from shielded skin in neonates receiving phototherapy: A prospective cohort study," Journal of Perinatology, vol. 37, no. 2, pp. 182-187, 2017. 


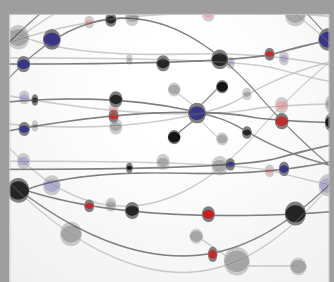

The Scientific World Journal
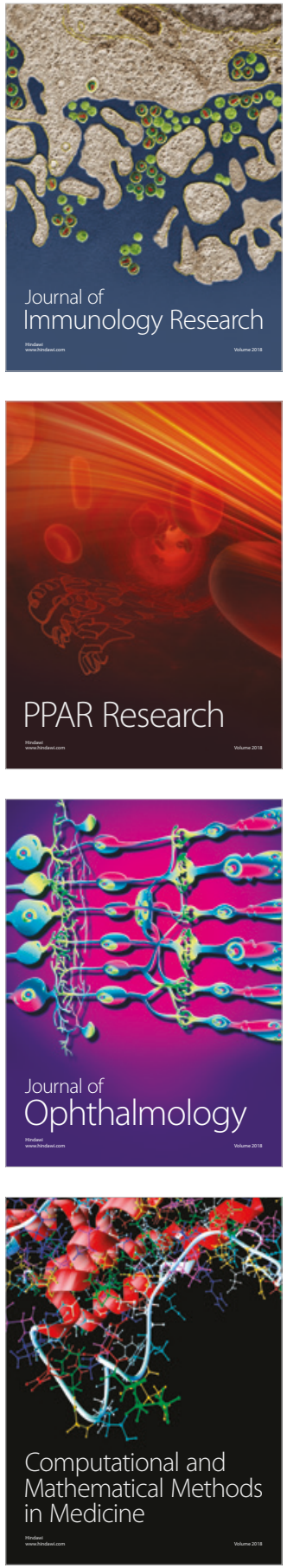

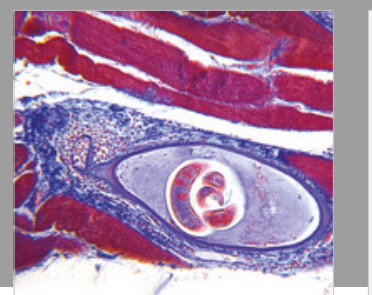

Gastroenterology Research and Practice

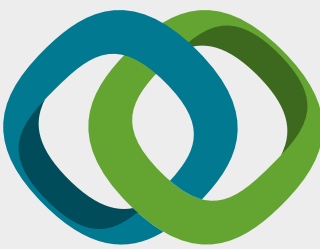

\section{Hindawi}

Submit your manuscripts at

www.hindawi.com
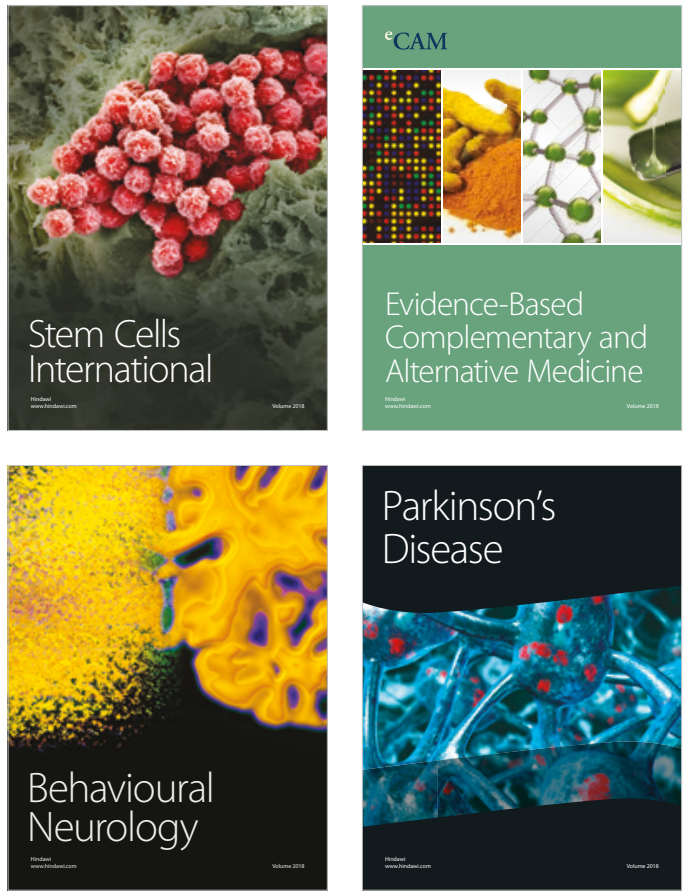

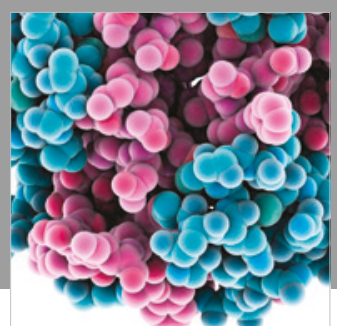

ournal of

Diabetes Research

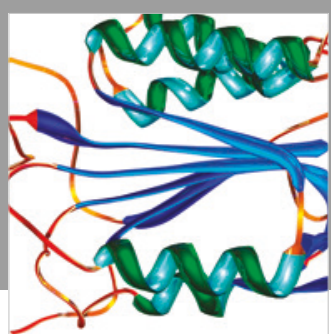

Disease Markers
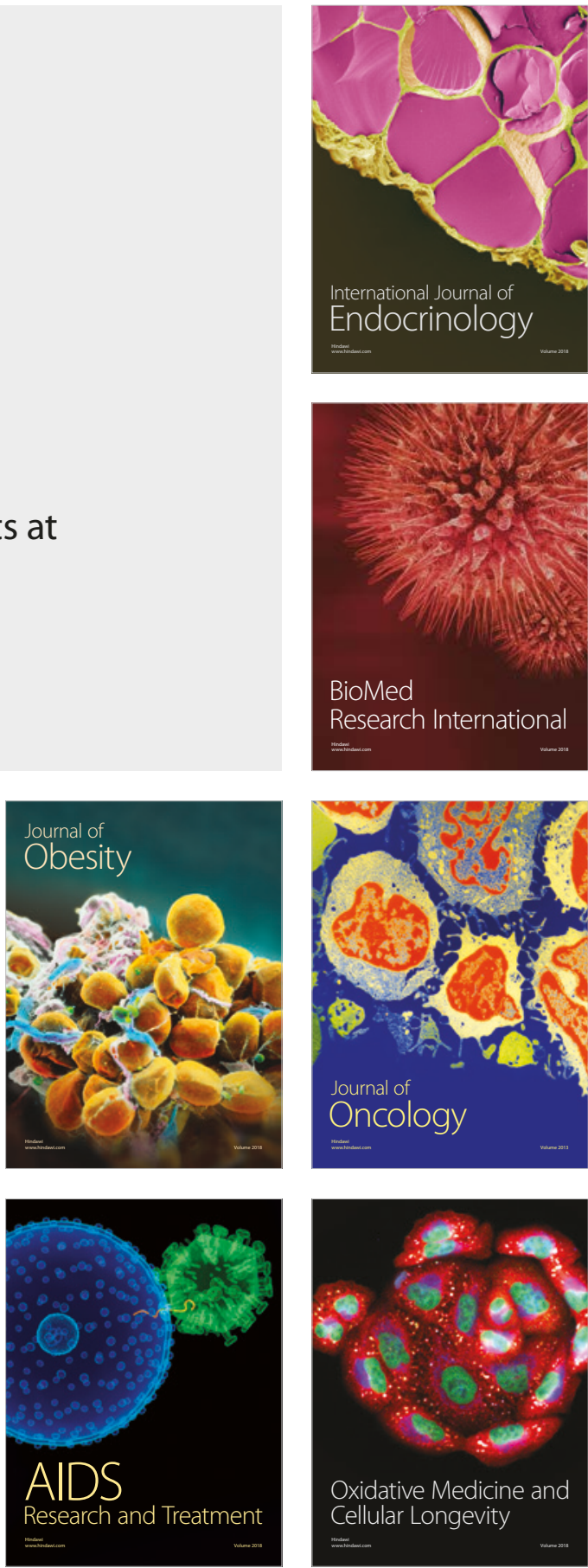